\title{
A novel hot-plate test sensitive to hyperalgesic stimuli and non-opioid analgesics
}

T.R. Lavich, R.S.B. Cordeiro, P.M.R. Silva and M.A. Martins
Laboratório de Inflamação, Departamento de Fisiologia e Farmacodinâmica, Instituto Oswaldo Cruz, FIOCRUZ, Rio de Janeiro, RJ, Brasil

\section{Correspondence \\ M.A. Martins \\ Departamento de Fisiologia e \\ Farmacodinâmica, FIOCRUZ \\ Av. Brasil, 4365 \\ 21045-900 Rio de Janeiro, RJ \\ Brasil \\ Fax: +55-21-2598-4395 \\ E-mail: mmartins@ioc.fiocruz.br \\ Research supported by CNPq and FAPERJ. T.R. Lavich \\ was the recipient of a \\ Doctoral fellowship from FAPERJ.}

Received March 29, 2004

Accepted December 14, 2004

\begin{abstract}
It is widely accepted that the classical constant-temperature hotplate test is insensitive to cyclooxygenase inhibitors. In the current study, we developed a variant of the hot-plate test procedure (modified hot-plate (MHP) test) to measure inflammatory nociception in freely moving rats and mice. Following left and right hind paw stimulation with a phlogogen and vehicle, respectively, the animals were placed individually on a hot-plate surface at $51^{\circ} \mathrm{C}$ and the withdrawal latency for each paw was determined simultaneously in measurements performed at $15,60,180$, and $360 \mathrm{~min}$ post-challenge. Plantar stimulation of rats $(250$ and $500 \mu \mathrm{g} / \mathrm{paw})$ and mice (125-500 $\mu \mathrm{g} / \mathrm{paw})$ with carrageenan led to a rapid hyperalgesic response of the ipsilateral paw that reached a plateau from 15 to 360 min after challenge. Pretreatment with indomethacin $(4 \mathrm{mg} / \mathrm{kg}, i p)$ inhibited the phenomenon at all the times analyzed. Similarly, plantar stimulation of rats and mice with prostaglandin $\mathrm{E}_{2}(0.5$ and $1 \mu \mathrm{g} /$ paw) also resulted in rapid hyperalgesia which was first detected 15 min post-challenge. Finally, we observed that the MHP test was more sensitive than the classical Hargreaves' test, being able to detect about 4- and 10-fold lower doses of prostaglandin $E_{2}$ and carrageenan, respectively. In conclusion, the MHP test is a simple and sensitive method for detecting peripheral hyperalgesia and analgesia in rats and mice. This test represents a low-cost alternative for the study of inflammatory pain in freely moving animals.
\end{abstract}

Key words

- Hyperalgesia

- Hargreaves' test

- Modified hot-plate test

- Carrageenan

- Prostaglandin $\mathrm{E}_{2}$

- Indomethacin

\section{Introduction}

It is well established that pain cannot be monitored directly in animals but can only be estimated by examining their responses $(1,2)$. Most of what is known about pain mechanisms is derived from rodent models of somatic nociception and hyperalgesia. Inflammatory algesimetric tests are based on the analysis of the overt behavioral responses directly triggered by the inflammatory process, as in models of abdominal constriction in mice (3) and rat joint incapacitation (4). Inflammation-dependent overt behavioral responses can also be indirectly triggered by an exogenous secondary stimulus, the most frequently used being either of a mechanical or thermal nature (1). 
In tests involving mechanical stimuli, it is the hind paw that is more frequently targeted. Inflammation is induced beforehand by subcutaneous administration of the phlogogen into the area to be stimulated with either constant pressure or gradually increasing pressure $(1,5)$. The nociceptive thresholds of the ipsilateral inflamed and contralateral healthy paw are then determined by the reflex withdrawal of the paw or by the appearance of a more complex behavioral response, such as the freezing reaction (6). Since the endpoint is a behavioral response, one potential problem concerning this type of tests is that the animals have to be restrained by the experimenter, a fact that may influence the results obtained.

In tests based on the use of thermal stimuli such as the hot plate and tail flick, the latency of nocifensive reactions evoked by heat stimuli of constant supra-threshold intensity $(7,8)$ or gradually increasing intensity (9) is measured. These methods are suitable for measuring the effects of opioid analgesics but they are not sensitive to the analgesic effects of nonsteroidal anti-inflammatory agents $(1,2,10)$. While trying to study hyperalgesia resulting from inflammation, Hargreaves et al. (11) developed a system in which radiant heat is applied to the plantar side of one hind paw inflamed by carrageenan, with the latency for paw withdrawal being measured with the help of a photoelectric-sensitive device. This is a test applied to unrestrained animals, most frequently rats, and which is sensitive to cyclooxygenase inhibitors.

More recently, Menendez et al. (12) modified the hot-plate system by developing the so-called unilateral hot-plate test, a method in which restrained mice have only one of their hind paw subjected to the heat stimulus each time. The test performed on the inflamed paw is reported to be sensitive enough to detect opiate analgesia as well as central and peripheral hyperalgesia. In order to avoid the putative influence of the restraint stress, we developed a variant of the constant-temperature hot-plate testing procedure for measuring inflammatory nociception in unrestrained rats and mice. A comparative analysis of sensitivity with the Hargreaves' radiant test was also carried out. Based on the simultaneous analysis of withdrawal latency of inflamed and non-inflamed hind paws of animals placed on a hot-plate surface, the modified hot-plate (MHP) test was shown to be a simple, rapid and sensitive method for pharmacological and inflammatory pain studies.

\section{Material and Methods}

\section{Animals}

All experiments were performed on male Wistar rats and Swiss mice of both sexes weighing 180 to $220 \mathrm{~g}$ and 20 to $25 \mathrm{~g}$, respectively. The animals were obtained from the breeding colony of the Oswaldo Cruz Foundation, Rio de Janeiro, RJ, Brazil, housed in groups of five and maintained on a 12-h light/dark cycle, with water and food ad libitum until use. The Ethics Committee for Care and Use of Laboratory Animals of the Oswaldo Cruz Foundation approved the experimental protocols employed in this study (License No. 0085-02).

\section{Stimuli and treatments}

Carrageenan (50-1000 $\mu \mathrm{g} / \mathrm{paw})$ or $\mathrm{PGE}_{2}$ (0.2-2 $\mu \mathrm{g} / \mathrm{paw})$ was injected intraplantarly (ipl) into the left hind paw whereas saline was injected into the right paw, both in a final volume of 100 and $50 \mu \mathrm{l}$ in rats and mice, respectively. Indomethacin (4 $\mathrm{mg} / \mathrm{kg}$ ) was administered intraperitoneally $1 \mathrm{~h}$ before the carrageenan challenge. Carrageenan was dissolved in sterile saline, whereas $\mathrm{PGE}_{2}$ was first dissolved in 10\% ethanol (stock solution) and further diluted with saline. Indomethacin was first dissolved in $0.2 \mathrm{~N} \mathrm{NaOH}$ followed by $\mathrm{pH}$ neutralization with Tris- $\mathrm{HCl}$ $(0.05 \%)$ and volume adjustment with saline. 
In all cases, control groups were treated with the solvent used for drug administration. All working solutions were prepared immediately before use.

Carrageenan, $\mathrm{PGE}_{2}$ and indomethacin were purchased from Sigma (St. Louis, MO, USA).

\section{Modified hot-plate test}

Animals were placed individually on a hot plate with the temperature adjusted to $51^{\circ} \mathrm{C}$ (Ugo Basile, Varese, Italy). Exposure to heat continued until nocifensive reaction of either hind paw occurred. The latency of the withdrawal response of each hind paw was determined at 15, 60, 180, and 360 min postchallenge. The left paw was stimulated with the phlogogenic agent (carrageenan or prostaglandin $\mathrm{E}_{2}, \mathrm{PGE}_{2}$ ) and the right one stimulated with sterile $0.9 \% \mathrm{NaCl}$ (saline) in a final volume of $100 \mu 1$. The heat source was maintained at constant intensity, which produced a stable withdrawal latency of approximately 8-10 $\mathrm{s}$ in vehicle-challenged paws. The animals were tested in only one series of measurements and the typical responses were hind paw shaking and/or lifting. The latency to the response was recorded manually with a chronometer, and the time of maximum permanence permitted on the hot surface was $20 \mathrm{~s}$. The experiments were performed in a sound-attenuated and air-conditioned $\left(20-22^{\circ} \mathrm{C}\right)$ laboratory. Hyperalgesia to heat was defined as a decrease in withdrawal latency and calculated as follows: $\Delta$ paw withdrawal latency $(\mathrm{s})=$ right paw withdrawal latency - left paw withdrawal latency.

\section{Hargreaves' plantar test}

The Hargreaves' test was performed as previously reported (11) using a standard apparatus (Ugo Basile). The test consisted of placing a rat in a transparent acrylic box and a mobile infrared heat lamp was positioned underneath the targeted hind paw. A thermal radiant stimulus was then applied to the plantar surface and the latency of the paw withdrawal response was measured automatically with the help of a photoelectricsensitive device. The latency of the withdrawal response of each hind paw was determined at $15,60,180$, and 360 min postchallenge, with the left paw being stimulated with the phlogogen (carrageenan or $\mathrm{PGE}_{2}$ ) and the right one stimulated with sterile saline in a final volume of $100 \mu \mathrm{l}$. Hyperalgesia in response to heat was defined as a decrease in withdrawal latency and calculated as follows: $\Delta$ paw withdrawal latency $(\mathrm{s})=$ right paw withdrawal latency - left paw withdrawal latency.

\section{Statistical analysis}

Data are reported as means \pm SEM and were analyzed statistically by one-way analysis of variance (ANOVA) followed by the Newman-Keuls-Student $t$-test. P values of 0.05 or less were considered to be significant.

\section{Results}

\section{Rat thermal hyperalgesia measured by the MHP test and sensitivity to indomethacin}

As illustrated in Figure 1, the ipl injection of carrageenan $(500 \mu \mathrm{g} / \mathrm{paw})$ into the left hind paw of rats caused a reduction in the latency of the withdrawal response to heat stimulation as compared to the right hind paw injected with vehicle. The $\Delta$ latency values, referred to from now on as hyperalgesic response, peaked 15 to $60 \mathrm{~min}$ postchallenge, decreasing thereafter, and were significant at 15, 60, 180, and $360 \mathrm{~min}$ after carrageenan stimulation as compared to $\Delta$ latency values for rats in which saline was injected into both the left and right hind paw (control group). The repeated application of the thermal test at these times did not change 
the $\Delta$ latency values as indicated by the reactivity of the control group (Figure 1).

Analysis of the analgesic effect of indomethacin was used to assess the sensitivity of the MHP test to cyclooxygenase inhibi-

Figure 1. Hyperalgesic effect of intraplantar injection of carrageenan $(500 \mu \mathrm{g} / \mathrm{paw}$; filled squares) into the left hind paw of rats, as measured by the modified hotplate test, and its sensitivity to indomethacin $(4 \mathrm{mg} / \mathrm{kg}$, ip; circles). Carrageenan stimulation was replaced with saline in the control group (open squares). Data are reported as means \pm SEM for at least 6 animals. ${ }^{+P}<$ 0.05 compared to saline-stimulated rats. ${ }^{*} P<0.05$ compared to carrageenan-untreated rats (one-way ANOVA followed by the Newman-Keuls test).

Figure 2. Hyperalgesic effect induced by intraplantar injection of carrageenan into the left hind paw of rats, as measured by the modified hot-plate test (A) and by the Hargreaves' test (B). Data are reported as means \pm SEM for at least 6 animals. ${ }^{*} \mathrm{P}<$ 0.05 compared to saline-stimulated rats (one-way ANOVA followed by the Newman-Keuls test).
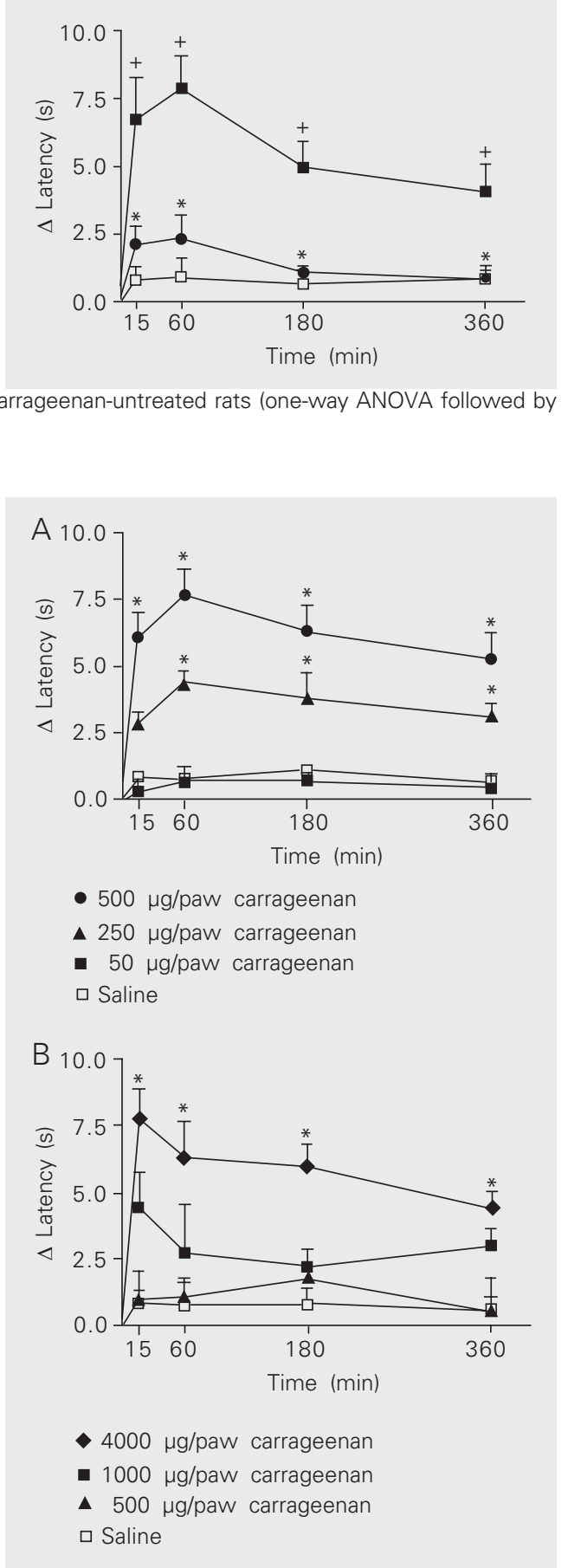

tors. As shown in Figure 1, treatment with indomethacin $(4 \mathrm{mg} / \mathrm{kg}$, ip $) 1 \mathrm{~h}$ before challenge prevented the carrageen-evoked hyperalgesic response at all the times analyzed.

Effect of carrageenan and $\mathrm{PGE}_{2}$ on rat paw withdrawal latencies measured by MHP and the Hargreaves' test

The hyperalgesic effect of increasing doses of carrageenan (50-4000 $\mu \mathrm{g} / \mathrm{paw})$ or $\mathrm{PGE}_{2}(0.25-1 \mu \mathrm{g} / \mathrm{paw})$ was measured using MHP and the Hargreaves' tests. As shown in Figure 2A, ipl injection of carrageenan into the rat hind paw at doses ranging from 50 to $500 \mu \mathrm{g} / \mathrm{paw}$ induced a dose-dependent hyperalgesic response as measured by the MHP test. The lowest effective dose of carrageenan able to induce a significant nocifensive reaction in the MHP test was $250 \mu \mathrm{g} / \mathrm{paw}$. However, when testing carrageenan-stimulated rats by the Hargreaves' method, statistically significant differences compared to control were obtained only with doses of $4000 \mu \mathrm{g} /$ paw (Figure 2B).

Assessed by either MHP or the Hargreave' test, the nocifensive response to $\mathrm{PGE}_{2}(0.25$ to $3 \mu \mathrm{g} / \mathrm{paw}$ ) also peaked from 15 to $60 \mathrm{~min}$, decreasing thereafter (Figure 3). Again the MHP test seemed to present greater sensitivity since the lowest effective dose, $0.5 \mu \mathrm{g} /$ paw, that induced a significant hyperalgesic response when tested by the MHP method (Figure 3A) was about four times lower than that measured by the Hargreaves' test (Figure 3B).

\section{Effect of carrageenan or $\mathrm{PGE}_{2}$ on paw} withdrawal latencies measured by the MHP test in mice

In mice, ipl administration of either carrageenan $(125-500 \mu \mathrm{g} /$ paw; Figure $4 \mathrm{~A})$ or $\mathrm{PGE}_{2}(0.5$ and $1 \mu \mathrm{g} / \mathrm{paw}$; Figure 4B) in a final volume of $50 \mu \mathrm{l}$ elicited a dose-dependent hyperalgesic response, as assessed by the MHP test. As noted for rats, the nocifensive 
response to both stimuli peaked from 15 to 60 min post-challenge, remaining significantly different from control for at least $6 \mathrm{~h}$.

\section{Discussion}

This study describes a variant of the classical hot-plate method suitable for measuring inflammatory nociception in freely moving rats and mice. The main issue dealt with by this paper is whether the hot-plate test, which has been classically employed for the measurement of supraspinally nociceptive integrated responses, could be adapted for detection of inflammatory pain and peripheral hyperalgesia. We found that after ipl injection of either carrageenan or $\mathrm{PGE}_{2}$, unrestrained rats and mice reacted to the thermal plantar stimulation with an acute decrease in the latency of the withdrawal response of the ipsilateral paw in comparison to the contralateral paw injected with vehicle. Hyperalgesic changes evoked by carrageenan detected in this MHP test were clearly sensitive to the cyclooxygenase inhibitor indomethacin. In addition, in comparison to the Hargreaves' method, the MHP test detected nocifensive responses at lower doses of carrageenan and $\mathrm{PGE}_{2}$. Taken together, these results suggest that the MHP test is a simple, rapid and sensitive method suitable for detecting peripheral hyperalgesia in unrestrained rats and mice.

It is well established that reflex latency reactions to thermal stimulation of non-inflamed paws using methods such as the classical hot-plate test are suitable for measuring the antinociceptive effects of opioid analgesics but are largely insensitive to nonsteroidal analgesics, including cyclooxygenase inhibitors $(1,2,10)$. However, using a modification of the hot-plate test, in which the plantar side of a single inflamed hind paw is placed on the hot-plate surface, Menendez et al. (12) demonstrated the usefulness of this method for detecting peripheral hyperalgesia in mice. It is worth mentioning that, to expose only one paw to the hot plate, the animals had to be restrained, a fact that may be considered a drawback of this method, since restraint stress alters analgesic and thermal effects by affecting central processing mechanisms of pain signaling (13).

These considerations make it reasonable to assume that a slight modification of the Menendez paw withdrawal test addressing the measurement of inflammatory pain in freely moving animals would be of some interest. In the present study, the latency of the paw withdrawal reflex following a constant-temperature plantar thermal stimulus was used as a measure of nociceptive response in unrestrained rats, which were challenged unilaterally with carrageenan in the hind paw. Our findings indicated that the threshold in the area of local tissue damage



- $1 \mu \mathrm{g} / \mathrm{paw} \mathrm{PGE}_{2}$

- $0.5 \mu \mathrm{g} /$ paw $P \mathrm{PE}_{2}$

$\triangle 0.25 \mu \mathrm{g} /$ paw $\mathrm{PGE}_{2}$

$\square$ Saline

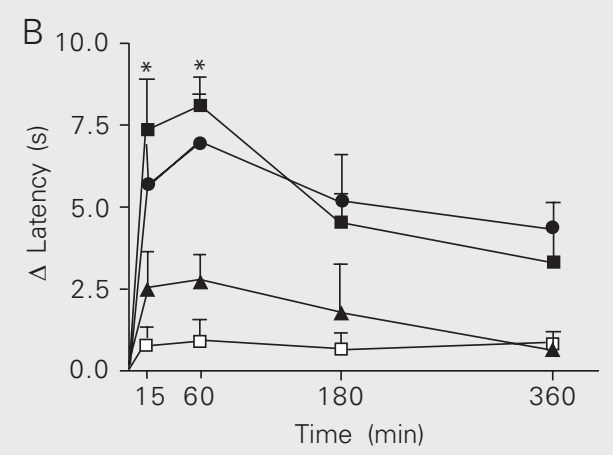

- $3 \mu \mathrm{g} / \mathrm{paw} \mathrm{PGE}_{2}$

- $2 \mu \mathrm{g} /$ paw $\mathrm{PGE}_{2}$

$\Delta 1 \mu \mathrm{g} / \mathrm{paw} \mathrm{PGE}_{2}$

$\square$ Saline
Figure 3. Hyperalgesic effect of intraplantar injection of prostaglandin $E_{2}\left(P_{G} E_{2}, 0.25-3 \mu g /\right.$ paw) into the left hind paw of rats, as measured by the modified hot-plate test (A) and the Hargreaves' test (B). Data are reported as means \pm SEM for at least 6 rats. ${ }^{*} P<0.05$ compared to saline-stimulated rats (one-way ANOVA followed by the Newman-Keuls test). 
created by the carrageenan challenge was about 5 to $7 \mathrm{~s}$ less than the threshold of the vehicle-treated contralateral paw. This indicates that the carrageenan challenge was capable of triggering a hyperalgesic response, which was very rapid in onset, peaking within 15 to 60 min post-challenge and remaining significant for at least $6 \mathrm{~h}$. Moreover, the ability to develop carrageenaninduced thermal hyperalgesia was completely absent in rats pretreated intraperitoneally with indomethacin. This agrees with the interpretation that the modified hot-plate test is suitable for the detection of peripheral hyperalgesia, as supported by our previous study using stimuli such as antigen, histamine, 5hydroxytryptamine or bradykinin (14).

In another set of experiments, we com-

Figure 4. Hyperalgesic effect of intraplantar injection of carrageenan (125-500 $\mu \mathrm{g} / \mathrm{paw}$; A) or prostaglandin $\mathrm{E}_{2}\left(\mathrm{PGE}_{2}, 0.5\right.$ and $1 \mu \mathrm{g} / \mathrm{paw}$; B) into the left hind paw of mice, as measured by the modified hot-plate test. Data are reported as means \pm SEM for at least 6 rats. ${ }^{*} \mathrm{P}<0.05$ compared to saline-stimulated mice (one-way ANOVA followed by the Newman-Keuls test).
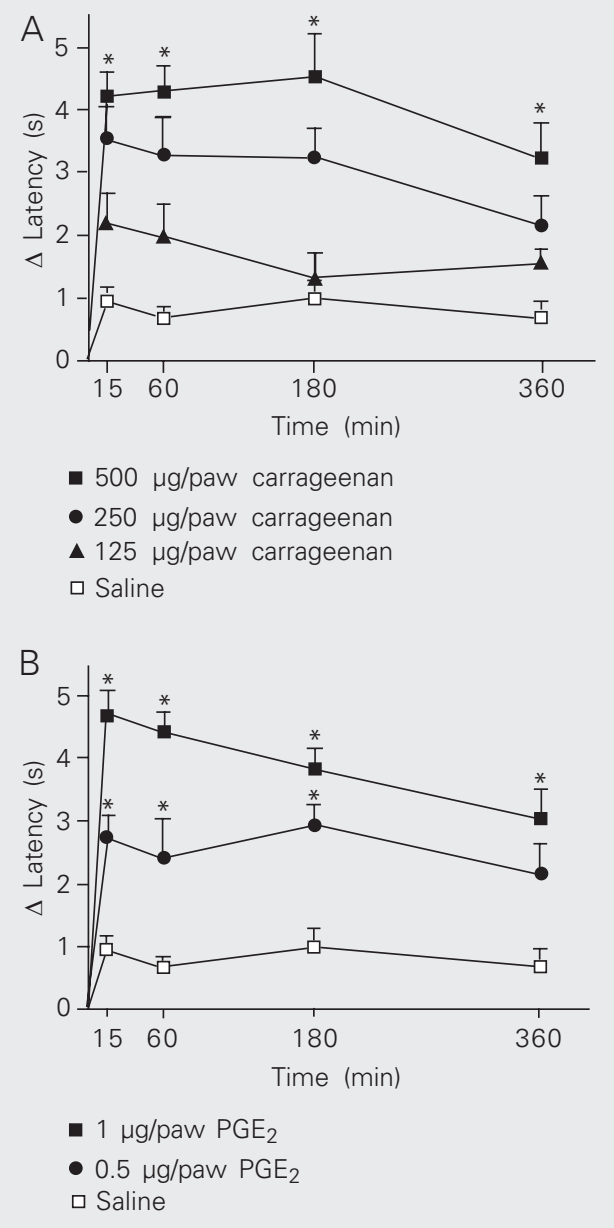

pared the effects of carrageenan and $\mathrm{PGE}_{2}$ on paw withdrawal latencies measured by MHP and the Hargreaves' thermal radiant focal test (11). We observed that both carrageenan and $\mathrm{PGE}_{2}$ exerted dose-dependent hyperalgesic effects as demonstrated by the two methods. However, the MHP test was more sensitive than the Hargreaves' test, being able to detect about 4- and 10-fold lower doses of $\mathrm{PGE}_{2}$ and carrageenan, respectively. The difference in contact area while using either the hot-plate device or the focal radiant stimulus may perhaps explain the large difference in sensitivity presented by these algometers, as also observed in previous studies $(12,15)$.

The hyperalgesic plantar effects of carrageenan and $\mathrm{PGE}_{2}$ were also assessed in mice. As observed following plantar provocation of rats, it was easy to detect a thermonocifensive response using the MHP method in mice whose hind paw was unilaterally stimulated with either carrageenan or $\mathrm{PGE}_{2}$. In both cases, a dose-dependent reduction in the withdrawal latency of the injected paw from $15 \mathrm{~min}$ to $6 \mathrm{~h}$ post-challenge was noted, indicating that the MHP test is equally useful when applied to mice. It is noteworthy that the hyperalgesic response to either carrageenan or $\mathrm{PGE}_{2}$ occurs later (mainly between the third and fourth hour post-challenge) in tests involving mechanical nociception applied to rats and mice $(16,17)$. Since mechanically insensitive C-fiber nociceptors able to respond to thermal stimuli have been identified (18), the possibility exists that activation of such nociceptors may contribute to the earlier response observed in the MHP test. In addition, one should not forget that heat provocation also stimulates thermoreceptors (1) and that, consequently, the dual activation of thermoreceptors and nociceptors may also cause quicker animal withdrawal from the thermal stimulus compared to the mechanical stimulus.

We propose that the MHP test is a rapid, sensitive and inexpensive method suitable for 


\section{detecting peripheral hyperalgesia in unre- tool in the study of pharmacological and strained rats and mice. Our findings thus inflammatory pain.} suggest that the MHP test may be a useful

\section{References}

1. Le Bars D, Gozariu M \& Cadden SW (2001). Animal models of nociception. Pharmacological Reviews, 53: 597-652.

2. Vogel HG \& Vogel WH (1997). Drug Discovery and Evaluation. Springer, Berlin/Heidelberg/New York.

3. Siegmund E, Cadmus R \& Lu G (1957). A method for evaluating both non-narcotic and narcotic analgesics. Proceedings of the Society for Experimental Biology and Medicine, 95: 729-731.

4. Tonussi CR \& Ferreira SH (1997). Bradykinin-induced knee joint incapacitation involves bradykinin B2 receptor-mediated hyperalgesia and bradykinin B1 receptor-mediated nociception. European Journal of Pharmacology, 326: 61-65.

5. Bianchi C \& Franceschini J (1954). Experimental observations on Haffner's method for testing analgesic drugs. British Journal of Pharmacology, 9: 280-284.

6. Ferreira SH, Lorenzetti BB \& Correa FM (1978). Central and peripheral antialgesic action of aspirin-like drugs. European Journal of Pharmacology, 53: 39-48.

7. D'Amour FE \& Smith DL (1941). A method for determination of pain sensation. Journal of Pharmacology and Experimental Therapeutics, 72: 74-79.

8. Woolfe G \& McDonald AD (1944). The evolution of the analgesic action of pethidine hydrochloride. Journal of Pharmacology and Experimental Therapeutics, 80: 300-307.

9. Hunskaar S, Berge OG \& Hole K (1986). A modified hot-plate test sensitive to mild analgesics. Behavioural Brain Research, 21: 101108.

10. Taber RI (1974). Predictive value of analgesic assays in mice and rats, in narcotic antagonists. In: Braude MC, Harris LS, May EL, Smith JP \& Villareal JE (Editors), Advances in Biochemical Psycho- pharmacology. Raven Press, New York.

11. Hargreaves K, Dubner R, Brown F, Flores C \& Joris J (1988). A new and sensitive method for measuring thermal nociception in cutaneous hyperalgesia. Pain, 32: 77-88.

12. Menendez L, Lastra A, Hidalgo A \& Baamonde A (2002). Unilateral hot-plate test: a simple and sensitive method for detecting central and peripheral hyperalgesia in mice. Journal of Neuroscience Methods, 113: 91-97.

13. Appelbaum BD \& Holtzman SG (1985). Restraint stress enhances morphine-induced analgesia in the rat without changing apparent affinity of receptor. Life Sciences, 36: 1069-1074.

14. Lavich TR, Cordeiro RS, Calixto JB, Silva PMR \& Martins MA (2003). Combined action of vasoactive amines and bradykinin mediates allergen-evoked thermal hyperalgesia in rats. European Journal of Pharmacology, 462: 185-192.

15. Vidulich L \& Mitchell D (2000). Responses of rats to noxious mechanical stimulation of their tails during tail reperfusion following transient ischaemia. Journal of Neuroscience Methods, 103: 173180.

16. Cunha TM, Verri Jr WA, Vivancos GG, Moreira IF, Reis S, Parada CA, Cunha FO \& Ferreira SH (2004). An electronic pressure-meter nociception paw test for mice. Brazilian Journal of Medical and Biological Research, 37: 401-407.

17. Vivancos GG, Verri Jr WA, Cunha TM, Schivo IR, Parada CA, Cunha FQ \& Ferreira SH (2004). An electronic pressure-meter nociception paw test for rats. Brazilian Journal of Medical and Biological Research, 37: 391-399.

18. Julius D \& Basbaum Al (2001). Molecular mechanisms of nociception. Nature, 413: 203-210. 